\title{
Translaminar Microendoscopic Herniotomy for Cranially Migrated Lumbar Disc Herniations Encroaching on the Exiting Nerve Root in the Preforaminal and Foraminal Zones
}

\author{
Ko Ikuta ${ }^{1}$, Osamu Tono ${ }^{2}$, Hideyuki Senba ${ }^{1}$, Takahiro Kitamura ${ }^{1}$, \\ Norihiro Komiya ${ }^{1}$, Masayoshi Oga ${ }^{3}$, Satoshi Shidahara ${ }^{1}$ \\ ${ }^{1}$ Department of Orthopedic Surgery, Karatsu Red Cross Hospital, Karatsu, Japan \\ ${ }^{2}$ Department of Orthopedic Surgery, Kyushu University Beppu Hospital, Beppu, Japan \\ ${ }^{3}$ Department of Orthopedic Surgery, Hiroshima Red Cross and Atomic-bomb Survivors Hospital, Hiroshima, Japan
}

\begin{abstract}
Study Design: Case series.
Purpose: The aim of this study was to describe translaminar microendoscopic herniotomy (TL-MEH) for cranially migrated lumbar disc herniations encroaching on the exiting nerve root in the preforaminal and foraminal zones and to report preliminary results of the procedure.

Overview of Literature: Conventional interlaminar approaches for preforaminal and foraminal lumbar disc herniations result in extensive removal of the lamina and facet joint to remove disc fragments safely. More destructive approaches increase the risk of postoperative segmental instability.

Methods: TL-MEH is a minimally invasive procedure for herniotomy via the translaminar approach using a microendoscopic technique. TL-MEH was performed in seven patients with a cranially migrated lumbar disc herniation encroaching on the exiting nerve root. The disc fragments were located in the preforaminal zone in four patients, and in the preforaminal and foraminal zones in three. Results: All patients experienced immediate relief from symptoms after surgery and satisfactory results at the final follow-up. Surgical complications, such as a dural tear, nerve injury, and surgical site infection, were not investigated.

Conclusions: TL-MEH seemed to be an effective and safe alternative minimally invasive surgical option for patients with a cranially migrated lumbar disc herniation encroaching the exiting nerve root in the preforaminal and foraminal zones.
\end{abstract}

Keywords: Lumbar disc herniation; Endoscopic surgery; Translaminar approach; Tubular surgery

\section{Introduction}

Macnab [1] introduced the term "hidden zone" in 1971 to define the lateral zone of the lumbar spine and indicated the difficulties related to exposing the lesion. The lateral zone has three sections, including the subarticular zone, the foraminal zone, and the extraforaminal zone. The foraminal zone is formed by the upper and lower pedicles, the dorsal aspect of the vertebral body, and the lateral edge of the interarticular portion of the lamina. In con-

Received May 14, 2012; Revised Aug 13, 2012; Accepted Aug 13, 2012

Corresponding author: Ko Ikuta

Department of Orthopedic Surgery, Karatsu Red Cross Hospital,

1-5-1 Futago, Karatsu 847-8588, Japan

Tel: +81-955-72-5111, Fax: +81-955-73-9530, E-mail: kou-ikuta@karatsu.jrc.or.jp 
trast, Bernucci and Giovanelli [2] defined a disc rupture that lies behind the posterior wall of the vertebra above and just medial to the medial margins of the pedicles as the "preforaminal herniated nucleus pulposus". In the present study, we used these distribution classifications for the lateral zone of the lumbar spine (Fig. 1).

The surgical approach to a preforaminal and foraminal disc herniation is controversial. The conventional interlaminar approach for these lesions results in extensive removal of the lamina and facet joint to remove the disc fragments safely. The more destructive approach of a wide bone resection to obtain better exposure increases the risk for postoperative segmental instability. Thus, a more

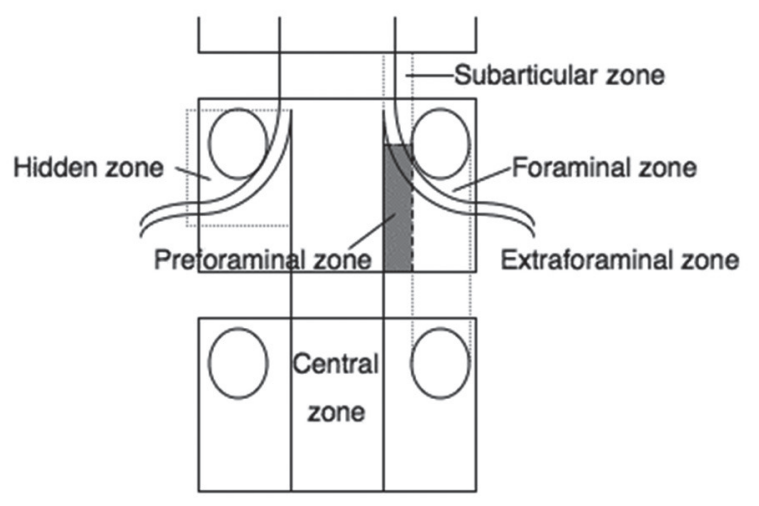

Fig. 1. Illustration showing the lateral zone classifications. The foraminal zone has two adjacent pedicles as its upper and lower boundaries. The preforaminal zone is just medial to the medial margins of the pedicles. direct approach for herniated discs was developed to prevent postoperative segmental instability in these cases. In 1998, Di Lorenzo et al. [3] described a pars interarticularis fenestration for foraminal lumbar disc herniation. They approached the foraminal disc herniation directly through a fenestration in the pars interarticularis. Soldner et al. [4] used a similar technique that they described as a "translaminar approach".

In contrast, minimally invasive procedures have been developed for spinal surgery in the last two decades. Microendoscopic discectomy (MED), which is an endoscopic technique that involves the use of a tubular retractor for surgical treatment of a lumbar disc herniation, is a minimally invasive procedure that was reported by Foley and Smith [5] in 1997. We have applied the MED technique for the translaminar approach since 2005 to make it a more minimally invasive procedure. The purpose of this study was to describe a translaminar microendoscopic herniotomy (TL-MEH) and report the preliminary results of the procedure.

\section{Materials and Methods}

A total of 115 patients with a lumbar disc herniation were surgically treated using the MED technique between January 2005 and December 2009. Seven (6\%) of these patients underwent TL-MEH for a cranially migrated lumbar disc herniation encroaching the exiting nerve root in the preforaminal and foraminal zones. The patient population consisted of five men and two women (mean age at the time of surgery, 66 years; range, 52-87 years).
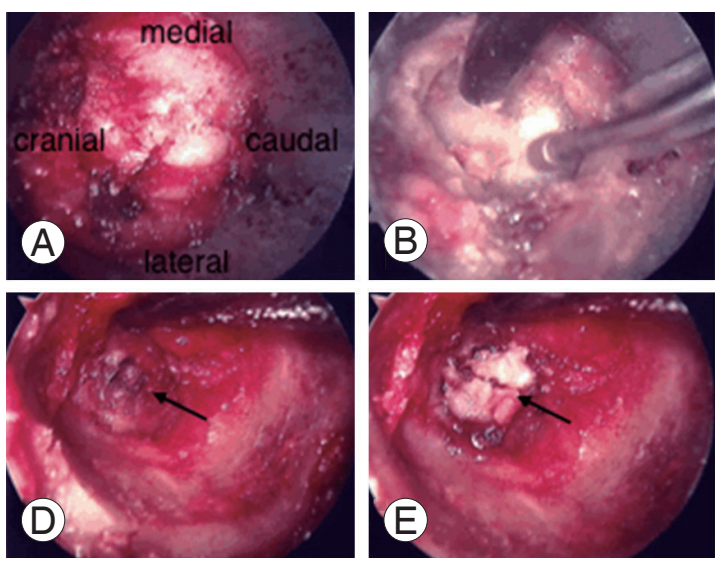
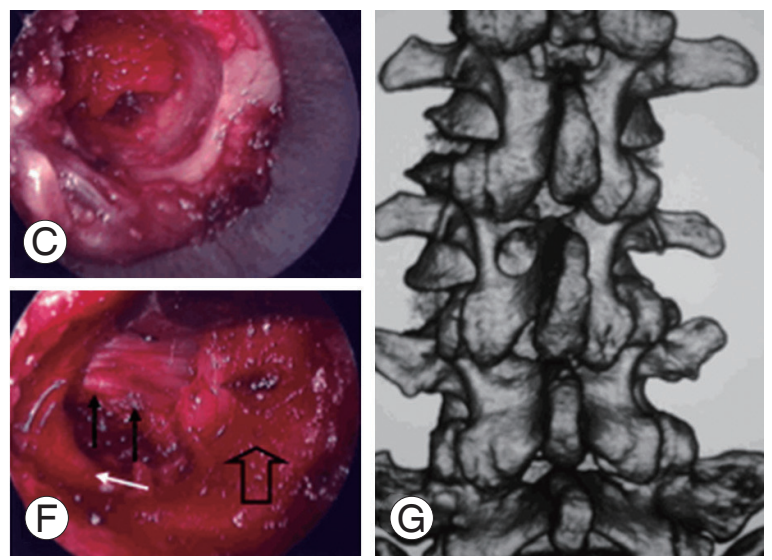

Fig. 2. Intraoperative photographs and a postoperative three-dimensional (3D) computed tomography (CT) scan of case 6. (A) A tubular retractor is placed on the targeted lamina. (B) An oval fenestration is made in the lamina using a high speed diamond drill. (C) The epidural space is exposed in the fenestration. (D) The preforaminal herniated disc (black arrow) is exposed. (E) Herniotomy is performed (black arrow). (F) The left L3 nerve root (white arrow) slipped caudally after decompression, and the posterior longitudinal ligament (black arrows) and the L3-4 disc (outlined arrow) were identified. (G) Postoperative 3D CT showing an oval fenestration in the left L3 lamina. 


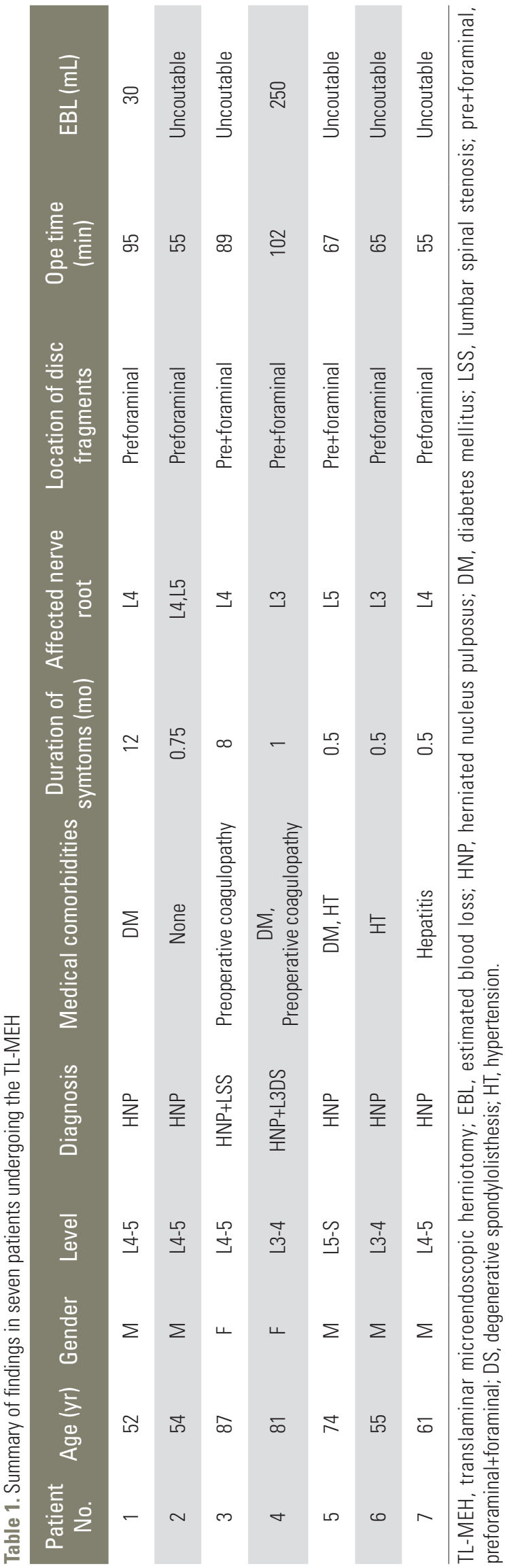

The affected disc level was L4-5 in four patients, L3-4 in two, and L5-S1 in one.

All patients complained of severe leg pain and low back pain, and had restrictions on daily activities. Average symptom duration was 3.3 months. Neurological examinations disclosed sensory impairment in the involved dermatome and motor weakness in all patients.

All patients underwent magnetic resonance imaging (MRI) of the lumbosacral spine before surgery. As a result, disc fragments were located in the preforaminal zone in four patients, and in the preforaminal and foraminal zones in three. The patient findings are summarized in Table 1.

Clinical outcome was evaluated using the Japanese Orthopedic Association (JOA) score for low back pain, and functional grade was assessed by Macnab's criteria [1].

\section{Surgical technique}

The procedure was performed with the patient in the prone position after induction of general anesthesia. The level of interest was identified using an image intensifier. A skin incision, approximately $2 \mathrm{~cm}$ in length, was made over the targeted lamina that covered the herniated disc. Serial dilators were passed to gently dilate the lumbar paraspinal muscles and to retract the lumbodorsal fascia. A 16-mm tubular retractor was then passed over the dilators and connected to a flexible-arm assembly mounted on the table. The tubular retractor was placed on the targeted lamina (Fig. 2A). After the dilators were removed, the endoscope was inserted into the tubular retractor and attached to the retractor.

After removing soft tissue from the bony surface, a small round or oval fenestration in the lamina was made using a high speed diamond drill to prevent injury to vascular and neural intraspinal structures (Fig. 2B, G). Although localization of the fenestration depended on the location of the herniated disc, care was taken to ensure that the fenestration spared at least $3 \mathrm{~mm}$ of the lateral border of the lamina to prevent an iatrogenic fracture of the pars interarticularis. In almost all cases, the ligamentum flavum ends cranially in this area, and the epidural space is often reached immediately (Fig. 2C).

The epidural exploration started along the lateral border of the thecal sac. Further dissection was directed towards the herniated disc fragments (Fig. 2D). The exiting nerve root is usually compressed upwards and backward 
Table 2. Clinical outcomes

\begin{tabular}{ccccc}
\multirow{2}{*}{$\begin{array}{c}\text { Patient } \\
\text { No. }\end{array}$} & Duration of F/U (mo) & \multicolumn{2}{c}{ JOA score (full marks: 29$)$} & \\
\cline { 3 - 4 } & & Preoperative & Postoperative & Assessment of the Macnab criteria \\
\hline 1 & 6 & 14 & 25 & Excellent \\
\hline 2 & 12 & 9 & 26 & Excellent \\
\hline 3 & 10 & 8 & 24 & Good \\
\hline 5 & 3 & 10 & 21 & Good \\
\hline 6 & 39 & 4 & 22 & Good \\
\hline 7 & 24 & 9 & 26 & Excellent \\
\hline
\end{tabular}

JOA, Japanese Orthopedic Association; F/U, follow-up.

by the herniated disc fragment. The lesion could be easily removed through the axillary portion of the exiting nerve root (Fig. 2E), and the root slipped caudally after decompression (Fig. 2F).

After inspecting the thecal sac and exiting nerve root, the site was copiously irrigated with physiological saline. The tubular retractor and endoscope were withdrawn, and a suction drain was left in the epidural space to prevent a postoperative epidural hematoma. The fascia and skin were closed routinely.

\section{Results}

Mean operative time was 76 minutes (range, 55-102 min-
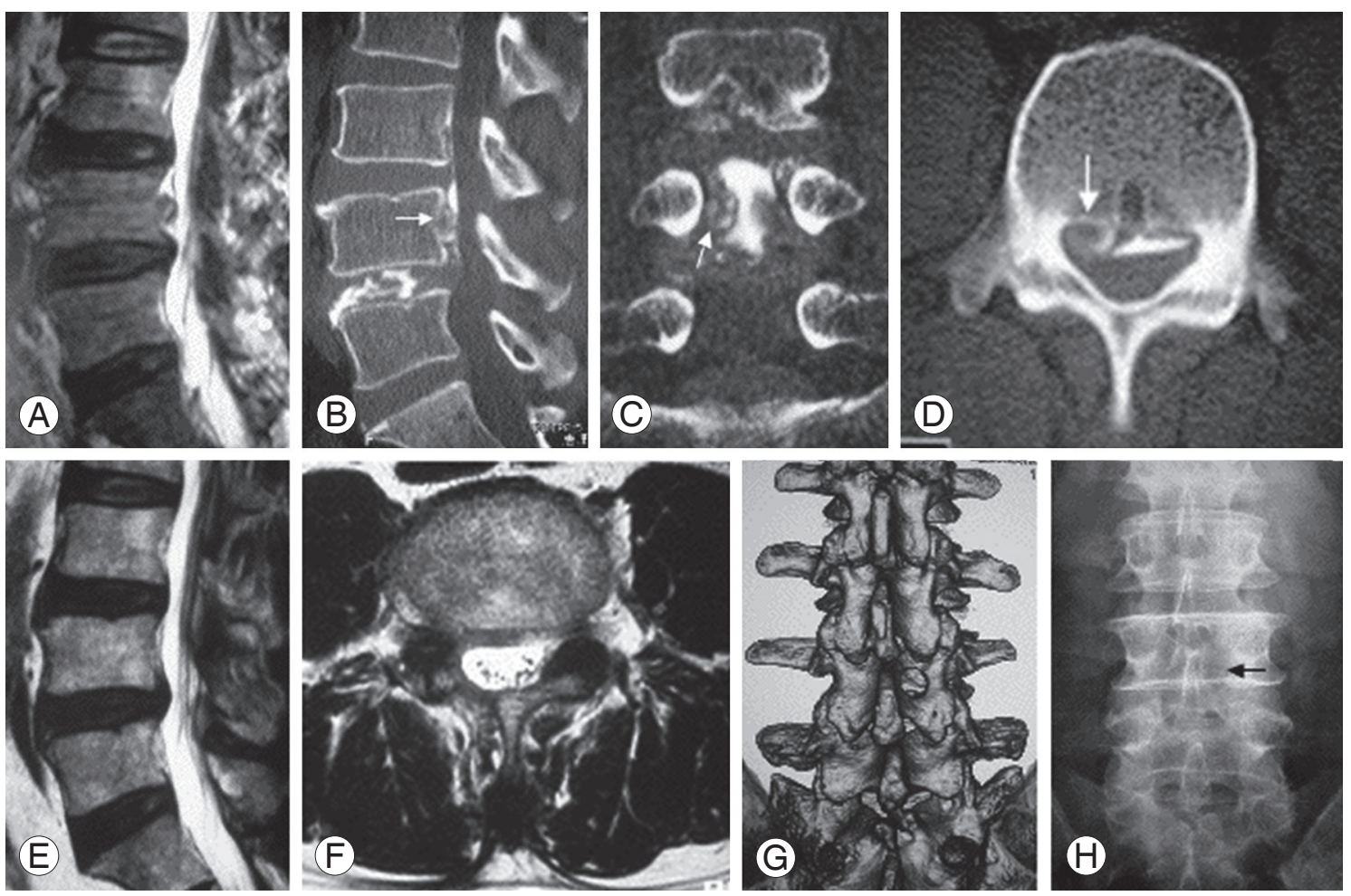

Fig. 3. Case 7. A 61-year-old man presented with a 2-week history of mild low back pain and severe pain radiating down the right lower extremity. (A) T2 weighted sagittal magnetic resonance image (MRI) showing a cranially migrated disc fragment behind the L4 vertebral body. (B-D) Computed tomography (CT) scans after discography demonstrated a cranially migrated disc fragment in the preforaminal zone (white arrows). (E, F) Sufficient decompression was confirmed on postoperative MRI. (G) Postoperative 3-dimensional CT scan showing an oval fenestration in the right L4 lamina. (H) A plain radiograph obtained 2 years after surgery shows a reduction in the size in the L4 lamina fenestration (black arrow). 
utes), and mean intraoperative estimated blood loss was $40 \mathrm{~mL}$ (range, uncountable-250 mL). Surgical complications such as fracture of the pars interarticularis, nerve injury, dural violation and cerebrospinal fluid leaks, and surgical site infection were not found in any patient.

Treatment was successful in all patients, with immediate pain relief and only mild postoperative discomfort. The follow-up examination was conducted after a mean interval of 14 months (range, 3-39 months), and improvement in clinical symptoms was maintained in all

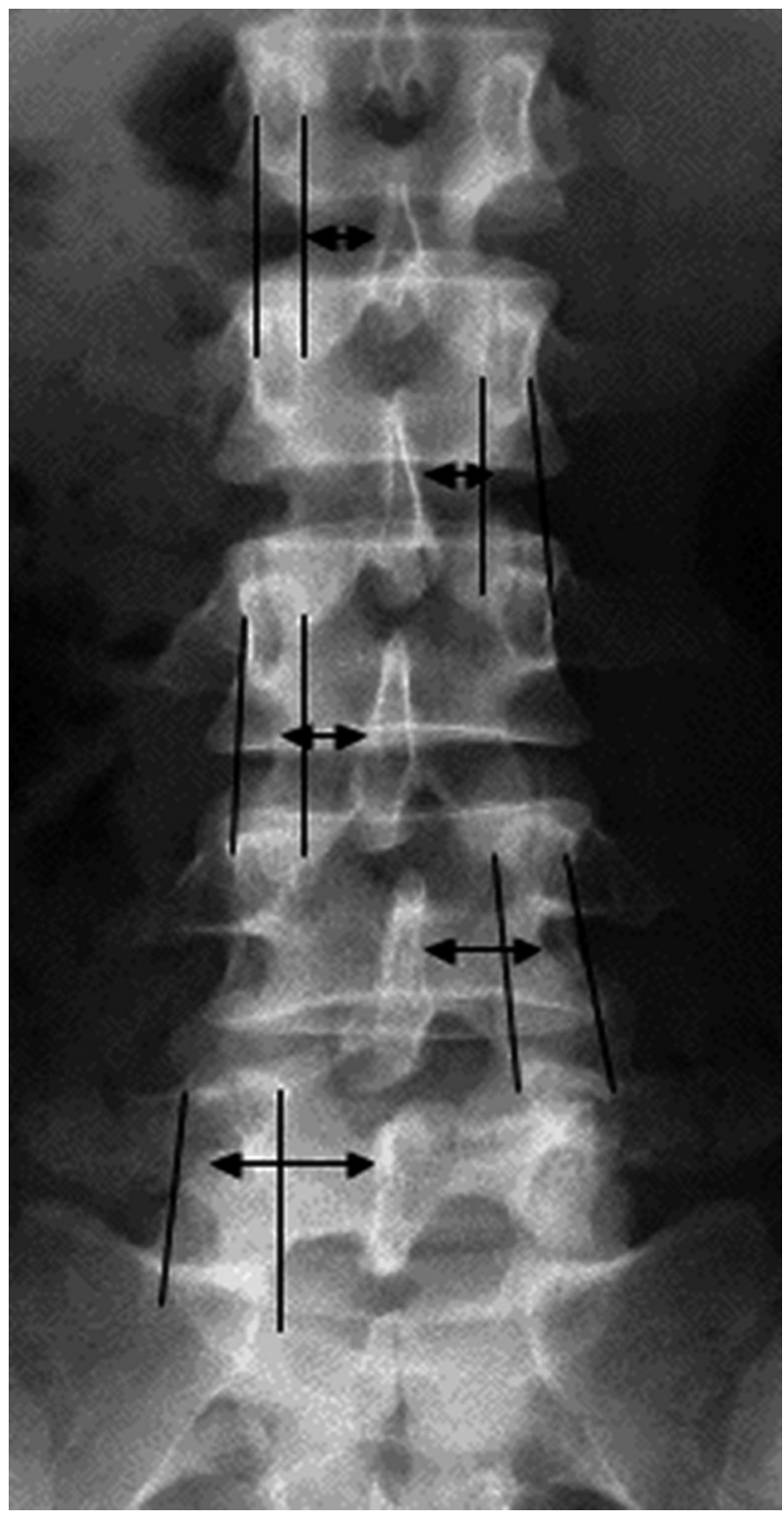

Fig. 4. Anatomical considerations. Isthmus lamina width of the lumbar spine (between black arrows) is progressively smaller at the more cranial levels, and the posterior structures of the foraminal zone (between black lines) vary at each level. patients. Mean JOA score (maximum score of 29 points) was 9 before surgery, and increased to 24.7 at the final follow-up. The functional grade according to Macnab's criteria was excellent in four patients and good in three. Clinical outcomes are summarized in Table 2 and an illustrative case (case 7) is shown in Fig. 3.

\section{Discussion}

The surgical approach for cranially migrated lumbar disc herniations in the preforaminal and foraminal zones is controversial. The conventional approach through the interlaminar space, such as a microsurgical approach described by Caspar et al. [6], proved to be unsuitable for preforaminal and foraminal disc herniations, because extensive removal of the lamina is necessary to remove the herniated disc fragments safely. The potential consequences of extensive removal of bone are a weakening of the pars interarticularis and development of postoperative segmental instability. Thus, a more direct approach to herniated discs was developed to prevent postoperative segmental instability in these cases. In 1998, Di Lorenzo et al. [3] described a pars interarticularis fenestration for foraminal lumbar disc herniation. They approached the foraminal disc herniation directly through a fenestration in the pars interarticularis. Soldner et al. [4] used a similar technique that they called the "translaminar approach". An advantage of the translaminar approach is that the exiting nerve root can be exposed with almost complete preservation of the facet joint and ligamentum flavum as opposed to the conventional interlaminar approach for preforaminal and foraminal lumbar disc herniations. Papavero et al. [7] reported a satisfactory clinical result using the translaminar approach in 104 patients with cephalad-extruded disc fragments impinging on the exiting root. They concluded that the translaminar approach is recommended for a disc herniation encroaching on the exiting root as an alternative to the conventional interlaminar route. Bernucci and Giovanelli [2] described a classic interlaminar interspace approach combined with a very limited translaminar fenestration for preforminal disc herniation and concluded that the microsurgical approach was safe and did not cause instability.

The width of the isthmus lamina of the lumbar spine is progressively smaller at more cranial levels, according to parameters measured by Reulen et al. [8] taken from 31 human cadavers. Accordingly, the posterior structures of 
the foraminal zone vary at each level. The intervertebral foramen at the cranial levels, particularly the levels above L2-3, has no posterior bony roof. In contrast, the foraminal zone has a posterior bony covering at the caudal levels. Consequently, lesions in the foraminal zone at levels below L3-4 can be exposed during the translaminar approach. In contrast, it is difficult to reach the affected disc and perform a discectomy through the fenestration in the lamina, particularly at L5-S1. However, we were able to reach the affected disc using the translaminar approach at the more cranial levels, because the lamina overlaps the disc space progressively at the more cranial levels (Fig. 4). Taken together, we prefer the use of the translaminar approach in patients with cranially migrated disc herniations in the preforaminal and foraminal zones at the L3-4 and L4-5 levels, and conditionally at the L5-S1 level if a discectomy is not necessary. This approach has been indicated for cranially migrated disc herniation in the preforaminal zone at $\mathrm{L} 2-3$.

Minimally invasive spinal surgery procedures have been developed over the last two decades. MED, which is an endoscopic technique that uses a tubular retractor for surgical treatment of a lumbar disc herniation, is a minimally invasive procedure [5]. TL-MEH is a minimally invasive procedure that applies the MED technique to a translaminar approach. The use of a tubular retractor during TL-MEH reduces surgical trauma, because the retractor is inserted after blunt dilatation of the paraspinal muscles, and it is not necessary to detach the multifidus muscles from the lower border of the lamina. Vogelsang [9] described the translaminar approach in 2007 in combination with a tubular retractor system, which is similar to the tubular retractor used during the MED technique, with a microscope for treating far cranio-laterally and foraminally extruded lumbar disc herniations. He concluded that the microscopic technique was reasonable and safe. As stated above, the -MEH offers several advantages such as a reduced tissue trauma and preservation of lumbar spine posterior structures, in comparison with the conventional approach through the interlaminar space. Furthermore, TL-MEH provides wide, illuminated, and excellent visualization, which increases surgical safety. In addition, lesions in the foraminal zone can be inspected beyond the fenestration during TL-MEH because the MED endoscope is angled at $25^{\circ}$. This is far superior to that of microscopic techniques.

\section{Conclusions}

TL-MEH was a safe and useful minimally invasive procedure for removing cranially migrated lumbar disc herniations encroaching the exiting nerve root in the preforaminal and foraminal zones and is a good alternative to the conventional interlaminar approach.

\section{Conflict of Interest}

No potential conflict of interest relevant to this article was reported.

\section{References}

1. Macnab I. Negative disc exploration: an analysis of the causes of nerve-root involvement in sixty-eight patients. J Bone Joint Surg Am 1971;53:891-903.

2. Bernucci C, Giovanelli M. Translaminar microsurgical approach for lumbar herniated nucleus pulposus (HNP) in the "hidden zone": clinical and radiologic results in a series of 24 patients. Spine (Phila Pa 1976) 2007;32:281-4.

3. Di Lorenzo N, Porta F, Onnis G, Cannas A, Arbau G, Maleci A. Pars interarticularis fenestration in the treatment of foraminal lumbar disc herniation: a further surgical approach. Neurosurgery 1998;42:87-9.

4. Soldner F, Hoelper BM, Wallenfang T, Behr R. The translaminar approach to canalicular and craniodorsolateral lumbar disc herniations. Acta Neurochir (Wien) 2002;144:315-20.

5. Foley KT, Smith MM. Microendoscopic discectomy. Tech Neurosurg 1997;3:301-7.

6. Caspar W, Campbell B, Barbier DD, Kretschmmer $\mathrm{R}$, Gotfried Y. The Caspar microsurgical discectomy and comparison with a conventional standard lumbar disc procedure. Neurosurgery 1991;28:78-86.

7. Papavero L, Langer N, Fritzsche E, Emami P, Westphal M, Kothe R. The translaminar approach to lumbar disc herniations impinging the exiting root. Neurosurgery 2008;62:173-7.

8. Reulen HJ, Muller A, Ebeling U. Microsurgical anatomy of the lateral approach to extraforaminal lumbar disc herniations. Neurosurgery 1996;39:345-50.

9. Vogelsang JP. The translaminar approach in combination with a tubular retractor system for the treatment of far cranio-laterally and foraminally extruded lumbar disc herniations. Zentralbl Neurochir 2007;68:24-8. 